\title{
Bottom Anti-Reflective Coatings (BARCs) for 157-nm Lithography
}

\author{
Liu He*, Rama Puligadda, Joyce Lowes, Michael Rich \\ Brewer Science, Inc., 2401 Brewer Drive, Rolla, MO 65401 USA
}

\begin{abstract}
Bottom anti-reflective coatings (BARCs) are essential for achieving the $65-\mathrm{nm}$ node resolution target by minimizing the substrate reflectivity to less than $1 \%$ and by planarizing substrates. We believe that the developments in 157-nm BARC products are on track to make them available for timely application in 157-nm lithography. We have made some significant improvements in resist compatibility and etch selectivity in relation to the latest available $157-\mathrm{nm}$ resists.

Two chromophores having desired high light absorbance at the 157-nm wavelength have been identified. The prototype BARC formulations basically meet the critical requirements for workable 157-nm BARCs, including optical properties, thermal stability, photo-stability, etch rate and selectivity, and compatibility with photoresists. The BARCs also show good coating quality and stripping resistance. Another essential feature of the BARCs is that they are formulated in industry-accepted safe solvents. The lithographic profiles of a benchmarked 157-nm photoresist on our prototype BARC LH157B show straight $60-\mathrm{nm} \mathrm{L/S}$ patterns. LH157B also exhibited excellent lithography performance as an ArF BARC. Optimization of the BARC formulations is in progress.
\end{abstract}

Keywords: $157 \mathrm{~nm}$, BARC, anti-reflective coating, lithography

\section{INTRODUCTION}

The most promising technology for the 65 -nm node size is still $157-\mathrm{nm}$ lithography because its integrated technology is almost ready for production. From a technical standpoint, 157-nm lithography currently has no competition that would preclude its use. It is well known that immersion 193-nm lithography currently shows promise as a solution for the industry's $65-\mathrm{nm}$ and $45-\mathrm{nm}$ chip production, and threatens to eclipse the upcoming 157-nm "dry" lithography generation prematurely, but the industry's $157-\mathrm{nm}$ program has made great progress and is in reasonably good shape. International Sematech (Austin, Texas) continues its 157-nm development coordination efforts. The Interuniversity Microelectronics Center (IMEC, Leuven, Belgium) recently installed the first full-field 157-nm stepper from ASML, and IMEC's work on 157-nm resists, pellicles, and BARCs is moving to a more mature stage. The resist suppliers have improved the 157-nm resists in transparency (optical density less than $1 / \square \mathrm{m}$ ) and etch resistance. Suppliers are confident that they can make photoresists available for the timely introduction of the $65-\mathrm{nm}$ and even smaller feature sizes for the 55-nm node. Birefringence of $\mathrm{CaF}_{2}$ for lenses is no longer a limitation, and $\mathrm{CaF}_{2}$ manufacturers are working on increasing the yield.

*Author to whom correspondence should be addressed. Telephone: 1-573-364-0300. Fax: 1-573-364-0650. E-mail: liu.he@brewerscience.com

Copyright 2004 Society of Photo-Optical Instrumentation Engineers. This paper will be published in Proceedings of SPIE: Advances in Resist Technology and Processing XXI, vol. 5376, John L. Sturtevant, editor, 2004, and is made available as an electronic preprint with permission of SPIE. One print or electronic copy may be made for personal use only. Systematic or multiple reproduction, distribution to multiple locations via electronic or other means, duplication of any material in this paper for a fee or for commercial purposes, or modification of the content of the paper are prohibited. 
The applications of i-line, DUV, and 193-nm lithography in industry have already proved that BARCs provide an innovative means to achieve dimensional control and are critical to the realization of greatly reduced feature sizes and application advances. The work presented in this paper also demonstrates that achieving good image quality and critical dimension control for the $65-\mathrm{nm}$ node size using $157-\mathrm{nm}$ lithography technology is not possible without the use of BARCs for minimizing the substrate reflectivity to less than $1 \%$ and for planarizing substrates.

In this paper, we present the latest 157-nm BARC developments at Brewer Science, Inc. Two chromophores having the desired high absorbance at the 157-nm wavelength have been identified. The prototype BARC formulation LH157B basically met the critical requirements for workable 157-nm BARCs, including those for optical properties, thermal stability, photo-stability, etch rate selectivity, and resist compatibility. The lithographic profiles of a benchmarked 157-nm photoresist on our BARCs show straight patterns down to $60-\mathrm{nm}$ L/S. LH157B also has appropriate optical constants at the 193-nm wavelength, and preliminary lithography results demonstrated that it also can be used as an ArF BARC.

\section{EXPERIMENTAL}

\subsection{Materials}

All chemicals used for synthesis of crosslinkable chromophoric polymers were reagent-grade products purchased from Aldrich, Fluka or Acros, and were used as received. Surfactants used for coating formulations were purchased from industry suppliers and were used as received.

\subsection{Synthesis}

After screening a variety of photochemicals, two compounds were identified as chromophores for 157-nm BARC formulations because these two compounds showed very strong light absorbance at the 157-nm wavelength. These two chromophores are denoted as Chromo-A and Chromo-B, respectively. Chromophoric polymers, in which Chromo-A or Chromo-B were chemically bonded to polymer backbone, were synthesized in the solvent propylene glycol monomethyl ether (PGME) in the presence of a base as the catalyst in an $\mathrm{N}_{2}$ atmosphere at an elevated temperature of between $100^{\circ}$ and $120^{\circ} \mathrm{C}$ for 20 to 24 hours. The resulting solution was used as the mother liquor for BARC coating solutions.

\subsection{Preparation of BARC formulations}

The BARC formulations were prepared by mixing the chromophoric polymer mother liquor, solvents, crosslinking agents, and catalysts to form a homogeneous solution. The solids content for the solution was adjusted to be $3 \%$ to $7 \%$ by weight for spin coating. The coating solution was deionized by stirring it with about $5 \%$ by weight ion exchange beads for 4 hours and then filtering it through a $0.10-\square \mathrm{m}$ pore size Teflon filter prior to spin application.

\subsection{Polymer molecular weight measurement}

Number-average molecular weight $(\mathrm{Mn})$, weight-average molecular weight $(\mathrm{Mw})$, and polydispersity index (PDI) of the chromophoric polymers in THF solutions were determined using gel permeation chromatography (GPC) on a Waters 717 plus Autosampler with RI detector (Waters 2410). The system was calibrated with respect to polystyrene standards.

\subsection{BARC thickness and refractive index $n$ and $k$ values}

Each BARC composition was applied onto three silicon wafers and cured. Thicknesses of the films were determined at multiple points on the three silicon wafers using a Gaertner ellipsometer and then averaged. The refractive index $\mathrm{n}$ and the extinction coefficient $\mathrm{k}$ were measured using a VUV-VASE spectroscopic ellipsometer (J.A. Woollam Co., Inc.).

\subsection{Solvent stripping test}

The stripping test is used routinely to determine whether the thermal curing step provides adequate BARC insolubilization to prevent removal by resist solvent. Ethyl lactate (a common resist solvent) was puddled on the cured BARC for 10 seconds. The wafers were then spun at $1500 \mathrm{rpm}$ for 15 seconds, and then at 
$3000 \mathrm{rpm}$ for 15 seconds. The film thickness of the BARC was then re-measured. If a thickness loss of more than $5 \%$ occurs, the product or cure cycle would likely be unacceptable.

\subsection{Exposure experiments}

BARC formulation LH157B was evaluated for lithographic performance with a 157-nm positive tone resist (Shipley XP0589) at SEMATECH. The resist was spin coated onto the 70-nm-thick BARC layer on 200$\mathrm{mm}$ bare silicon wafers. The resist thickness was $170 \mathrm{~nm}$, its post-application bake (PAB) conditions were $100^{\circ} \mathrm{C}$ for 60 seconds, and its post-etch bake (PEB) conditions were $110^{\circ} \mathrm{C}$ for 60 seconds. Dense and isolated line features ranging in size from 50 to $80 \mathrm{~nm}$ were patterned using a $157-\mathrm{nm}$ laser microstepper (numerical aperture: 0.85 , sigma: 0.3 ) with an alternating phase-shift mask (alt-PSM). The exposed resist was developed in the $2.38 \%$ TMAH solution. The lithographic profiles were examined in cross-section with a scanning electron microscope (SEM).

\subsection{Storage stability}

The BARC LH157B was deionized by 650C ion exchange beads for 4 hours, and the beads were removed by straining the solution through plastic cloth. The solution was then filtered through a $0.1-\square \mathrm{m}$ pore size Teflon filter into $60-\mathrm{ml}$ screw-cap Nalgene bottles. Freshly opened bottles were used when measuring the effects of aging on film thickness. The coating quality was checked under a microscope. Spin and cure conditions remained constant throughout the test.

\section{RESULTS AND DISCUSSION}

\subsection{Chromophore screening}

Refractive indices $\mathrm{n}$ (real) and $\mathrm{k}$ (imaginary, also called the extinction coefficient) of a BARC are optical constants that indicate the BARC's light absorbance and reflectivity control on a given substrate and underneath a given photoresist. Prolith modeling software was used to determine the optimum $\mathrm{n}$ and $\mathrm{k}$ values for 157-nm BARCs. By analyzing contour plots, it was found that the refractive index $n$ (real) of 1.4 to 1.8 and $k$ (imaginary) of 0.3 to 0.6 (which corresponds to an optical density (OD) of greater than or equal to $10.4 \square \mathrm{m}^{-1}$ at $157-\mathrm{nm}$ wavelength) are applicable and optimum ranges for a BARC to reduce substrate reflectivity to less than $1 \%$. These ranges were chosen as targets for the screening of materials for $157-\mathrm{nm}$ BARCs.

A variety of photochemical compounds were blended with a Cymel resin in PGME for chromophore screening. The Cymel resin was used as a model polymer for preparing high-quality films, which were used for $\mathrm{n}$ and $\mathrm{k}$ measurement. Two chromophores, Chromo-A and Chromo-B, were selected for 157-nm BARC formulation because the films containing Chromo-A or Chromo-B had strong light absorbance at the 157-nm wavelength.

Table 1. Optical constants and properties of Chromo-A and Chromo-B.

\begin{tabular}{c|c|c|c}
\hline $\begin{array}{c}\text { Chromophore } \\
\text { contained in the film }\end{array}$ & $\begin{array}{c}\mathrm{n} \text { and } \mathrm{k} \text { at } \\
157 \mathrm{~nm}\end{array}$ & Good solvent(s) & $\begin{array}{c}\text { Possible to chemically bond to } \\
\text { polymer backbone? }\end{array}$ \\
\hline Chromo-A & $\mathrm{n}=1.62 ; \mathrm{k}=0.50$ & PGME & Yes \\
\hline Chromo-B & $\mathrm{n}=1.65 ; \mathrm{k}=0.45$ & Industry solvents & Yes \\
\hline
\end{tabular}

As shown in Table 1, both Chromo-A and Chromo-B gave desired $\mathrm{n}$ and $\mathrm{k}$ values. Figure 1(a) shows the reflectance curve of $157-\mathrm{nm}$ resist XP0589 (at a thickness of 150 to $200 \mathrm{~nm}$ ) on a BARC containing Chromo-A ( $\mathrm{n}=1.62, \mathrm{k}=0.50)$, and Figure 1(b) shows the reflectance curve of a BARC containing Chromo-B $(\mathrm{n}=1.65, \mathrm{k}=0.45)$. For Chromo-A, the first minimum $(27 \mathrm{~nm})$ of BARC thickness can reduce the substrate reflectivity to less than $1 \%$ because of the BARC's high k value. For Chromo-B, the first minimum $(27 \mathrm{~nm})$ of BARC thickness results in $1.6 \%$ reflectivity, and the second minimum $(70 \mathrm{~nm})$ of BARC thickness can reduce the reflectivity to less than $1 \%$. 
Of the two BARC candidates we selected the polymer with attached Chromo-B for further development for two reasons. First, the first minimum thickness for the BARC containing Chromo-A ( $27 \mathrm{~nm})$ may be too thin to planarize topography on the substrate. Second, Chromo-A has marginal solubility in solvents other than PGME, which leads to a low flash point for the formulation. In contrast, Chromo-B is soluble in industry-accepted solvents and can be readily grafted to the polymer backbone. The second minimum thickness of $70 \mathrm{~nm}$ obtained with the Chromo-B formulation is suitable for planarizing the substrate. Lithography testing for the BARC containing Chromo-B (LH157B) was done using a film thickness of 70 nm.

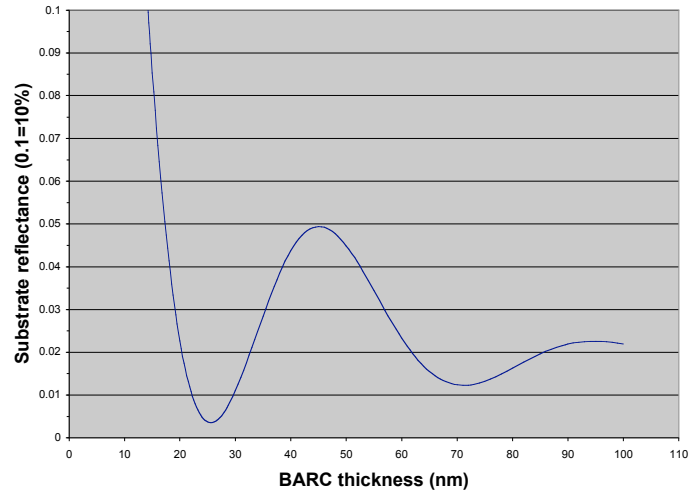

(a)

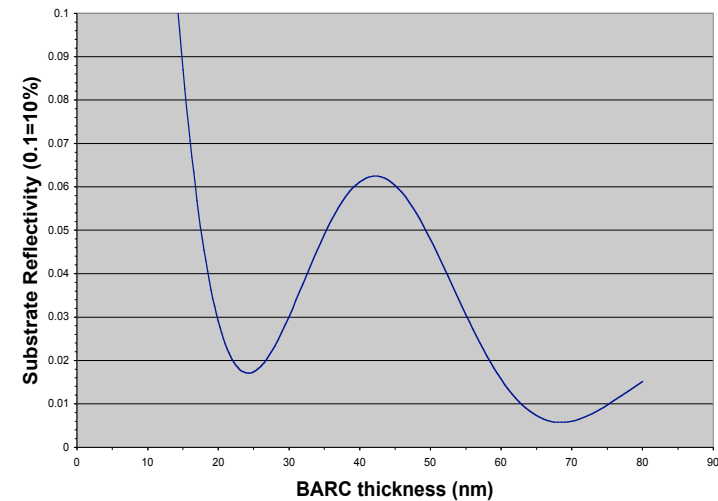

(b)

Figure 1. Reflectivity curves with 157-nm resist XP0589, silicon as substrate, and (a) BARC containing Chromo-A or (b) BARC containing Chromo-B.

\subsection{Attachment of Chromo-B to polymer backbone}

A polymer was identified for 157-nm BARC formulation. This polymer has the desired features to meet the critical requirements for $157-\mathrm{nm}$ BARC design ${ }^{[1]}$. The polymer is stable upon exposure to a $157-\mathrm{nm}$ laser, shows fast etch rate, has good solubility in industry-accepted solvents (PGME, PGMEA, etc.), and exhibits high spin-bowl compatibility. Another important feature is that it has pendant functional groups, which can be used to attach Chromo-B to its backbone.

In order to confirm that Chromo-B can be consistently attached to the polymer during synthesis, five labscale batches of polymers with attached Chromo-B were synthesized. These batches were used as mother liquors for BARC formulation LH157B. Their molecular weights were measured by GPC. Figure 2 shows the GPC profiles for each of the mother liquors.

From Figure 2, it can be seen that the profiles for five batches of polymers with attached Chromo-B are identical and the elution times for each polymer are the same, which indicates that the polymers with attached Chromo-B have the same molecular weight and polydispersity index. These results mean the synthesis procedure for attaching Chromo-B to the polymer backbone is reproducible and repeatable.

\subsection{Lithography performance}

The critical requirements for 157-nm BARCs ${ }^{[1]}$ were achieved by the prototype formulation LH157B. After the films were baked at $180^{\circ}$ to $205^{\circ} \mathrm{C}$ for 60 seconds, the films had no thickness change after stripping in ethyl lactate. The coating quality was excellent. The storage life for the formulation was longer than 4 months at room temperature. LH157B was then submitted to SEMATECH for lithography performance evaluation. The lithography performance of 157-nm resist XP0589 on LH157B was evaluated. The resist thickness was $170 \mathrm{~nm}$, and according to the reflectivity curve obtained by Prolith modeling, the 
second minimum BARC thickness of $70 \mathrm{~nm}$ was applied to keep the substrate reflectivity at less than $1 \%$. See Figure 1(b).

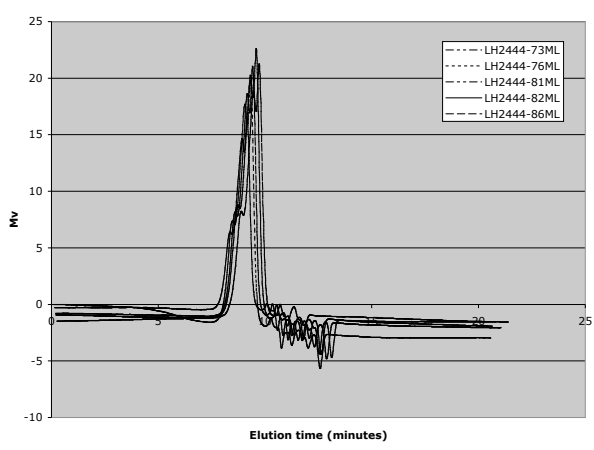

Figure 2. GPC profiles of LH157B mother liquors for lab-scale synthesis consistency test.

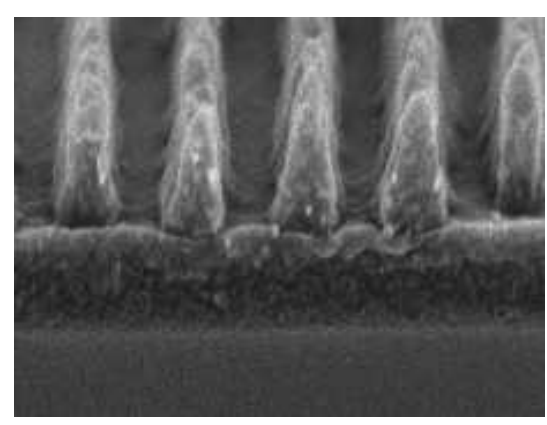

Figure 3. Lithographic profile of resist XP0589 on a benchmarked ArF BARC, $90 \mathrm{~nm}$, L/S 1:1 (courtesy of SEMATECH).

As a comparison, the lithography performance of resist XP0589 with one benchmarked ArF BARC was also evaluated. Figure 3 shows the profile $(90 \mathrm{~nm}, \mathrm{~L} / \mathrm{S} \mathrm{1:1)}$ of XP0589 on the ArF BARC.

From Figure 3, footing and scumming are obvious in the profile. The benchmarked ArF BARC definitely was not suitable for application as a 157-nm BARC.

Figure 4 shows profiles of XP0589 on the 157-nm BARC LH157B. From Figures 3 and Figure 4, it is very clear that 157-nm BARC LH157B can significantly improve the resist pattern. LH157B enables the XP0589 resist to achieve $60 \mathrm{~nm}, \mathrm{~L} / \mathrm{S} \mathrm{1:1}$ and $55 \mathrm{~nm}, \mathrm{~L} / \mathrm{S} \mathrm{1:1.5}$ resolution. The pattern shape is straight, and no footing, scumming, or undercutting is observed.

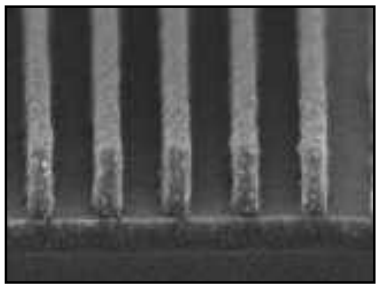

(a) $80 \mathrm{~nm} 1: 1.5$

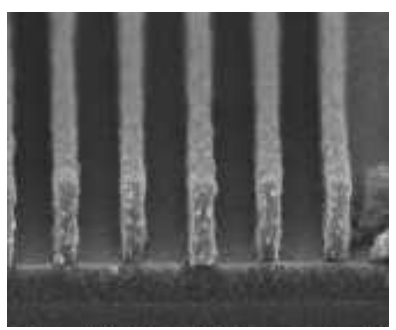

(d) $60 \mathrm{~nm} \mathrm{1:1.5}$

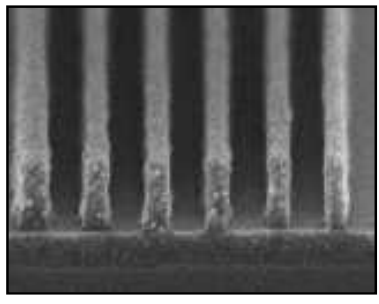

(b) $70 \mathrm{~nm} \mathrm{1:1}$

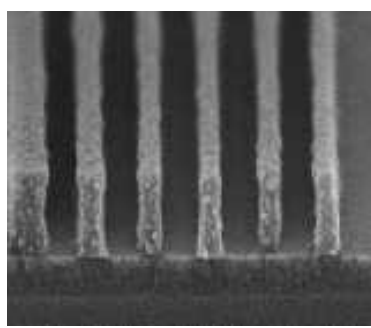

(e) $55 \mathrm{~nm} \mathrm{1:1.5}$

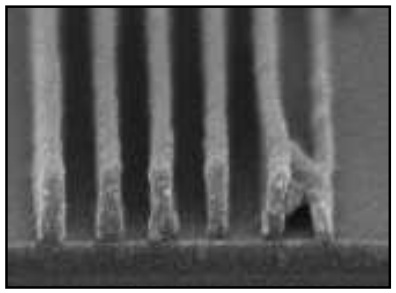

(c) $60 \mathrm{~nm} \mathrm{1:1}$

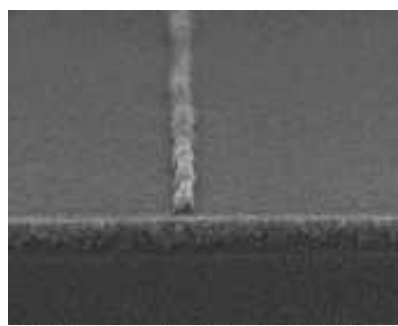

(f) $55 \mathrm{~nm}$ iso

Figure 4. Lithographic profiles of XP0589 resist on the 157-nm BARC LH157B. Resist thickness=170 nm; BARC thickness $=70 \mathrm{~nm}$. Alt-PSM mask., $\mathrm{NA}=0.85, \square=0.3$. PAB: $100^{\circ} \mathrm{C} / 60 \mathrm{sec} ; \mathrm{PEB}: 110^{\circ} \mathrm{C} / 60 \mathrm{sec}$. 
After the lithography process was optimized, the profiles of the XP0589 resist on BARC LH157B were

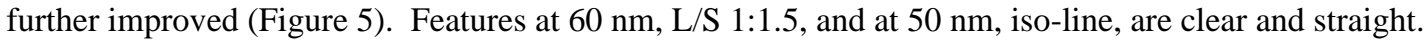

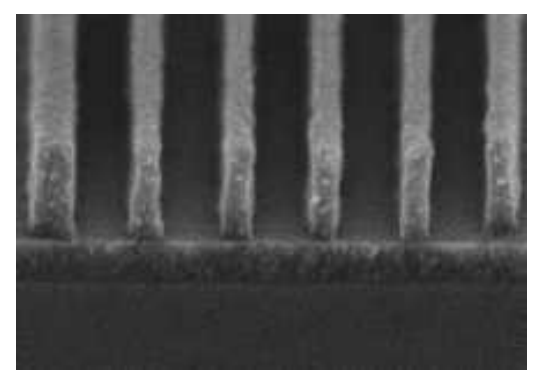

(a) $60 \mathrm{~nm} 1: 1.5$

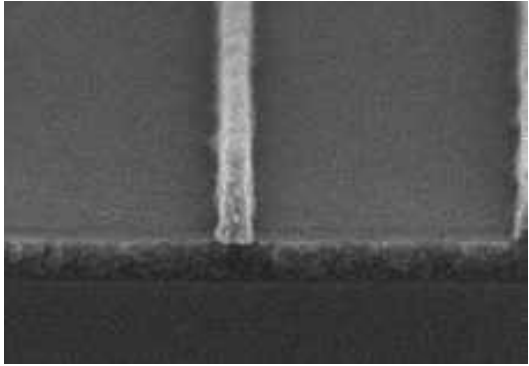

(b) $50 \mathrm{~nm}$ Iso-line

Figure 5. Lithographic profiles of XP0589 resist on the 157-nm BARC LH157B. (a) $60 \mathrm{~nm}, \mathrm{~L} / \mathrm{S} \mathrm{1;1.5;} \mathrm{(b)} 50 \mathrm{~nm}$, iso line. Resist thickness $=170 \mathrm{~nm}$; BARC thickness $=70 \mathrm{~nm}$.

In addition to its fast etch rate, another important feature of LH157B is its refractive indices $\mathrm{n}$ and $\mathrm{k}$ at 193nm wavelength: $\mathrm{n}=1.70$ and $\mathrm{k}=0.38$, which are suitable for ArF BARC formulation. LH157B was thus expected to be used as an ArF BARC. The lithography performance of ArF resist GAR 8150G1 with LH157B was evaluated at IMEC. The thickness of the GAR8150G1 resist was $270 \mathrm{~nm}$, its PAB and PEB conditions were $115^{\circ} \mathrm{C}$ for $90 \mathrm{sec}$, the BARC thickness was $80 \mathrm{~nm}$, and the baking conditions were $205^{\circ} \mathrm{C}$ for $60 \mathrm{sec}$.

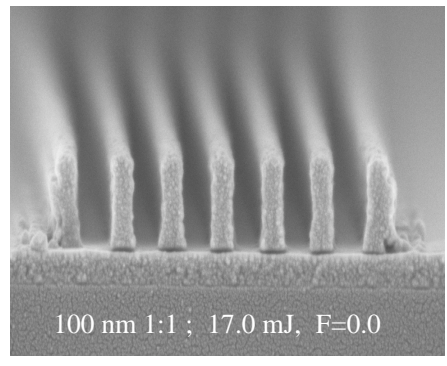

(a)

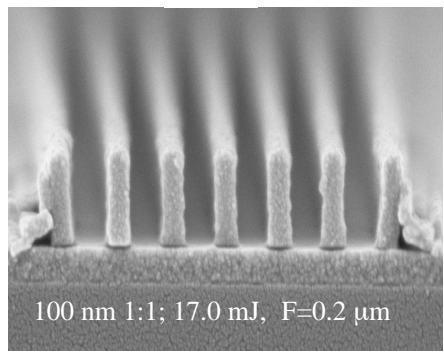

(c )

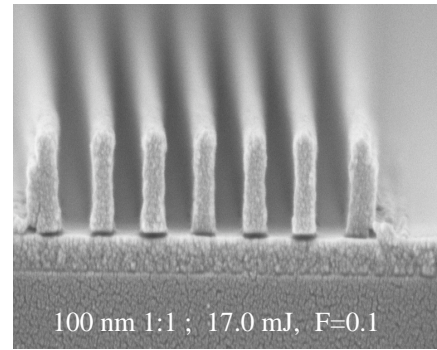

(b)

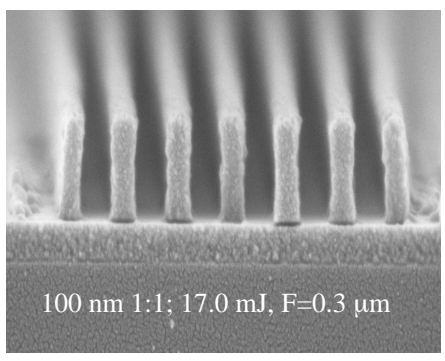

(d)

Figure 6. Lithographic profiles of ArF resist GAR8105G1 on BARC LH157B. (a) $100 \mathrm{~nm}, \mathrm{~L} / \mathrm{S} 1: 1, \mathrm{~F}=0.0 \square \mathrm{m}$;

(b) $100 \mathrm{~nm}, \mathrm{~L} / \mathrm{S} 1: 1, \mathrm{~F}=0.1 \square \mathrm{m}$; (c) $100 \mathrm{~nm}, \mathrm{~L} / \mathrm{S} 1: 1, \mathrm{~F}=0.2 \square \mathrm{m}$; and (d) $100 \mathrm{~nm}, \mathrm{~L} / \mathrm{S} 1: 1, \mathrm{~F}=0.3 \square \mathrm{m}$. Resist thickness $=270 \mathrm{~nm}$; BARC thickness $=80 \mathrm{~nm}$.

The depth of focus varied from 0.0 to $0.3 \square \mathrm{m}$, the $100 \mathrm{~nm}, \mathrm{~L} / \mathrm{S} 1: 1$ resolution is achieved, the pattern shape is straight, and no footing or undercutting are observed. The results in Figure 6 show that LH157B also can 
be used as an ArF BARC after modification.

\section{CONCLUSIONS}

Two chromophore compounds, Chromo-A and Chromo-B, have been identified for use in 157-nm BARC formulations due to their strong light absorbance at the 157-nm wavelength. Prototype BARC LH157B was made from a polymer with attached Chromo-B and exhibited features meeting industry requirements for 157-nm BARCs. The combination of resist XP0589 on LH157B has shown excellent lithography performance, which significantly improved over original benchmarking results at SEMATECH. LH157B can be applied as a BARC for 157-nm lithography technology.

Because LH157B also has the features desired for ArF BARCs, the preliminary lithography results of ArF resist GAR8105G1 with LH157B are very promising. LH157B is expected to be used as an ArF BARC after formulation modification. The optimization is in progress.

\section{REFERENCE}

[1] Liu He, Rama Puligadda, Joyce Lowes, Michael Rich, "Bottom Anti-Reflective Coatings (BARCs) for 157-nm Lithography,” Proceedings of SPIE, vol. 5040, 2003, pp.1386-1395.

\section{ACKNOWLEDGMENTS}

This material is partially based upon work supported by the National Science Foundation under Grant No. 0319158.

The authors would like to thank Georgia Rich and Karen Turnquest of International SEMATECH for their 157-nm lithography work. The authors also would like to thank Mariya Nagatkina (Brewer Science assignee to IMEC) for 193-nm lithography work. 